\title{
Influence of elongation and washing on double-layer R2R-printed flexible electrodes for smart clothing applications
}

\author{
Rafal Sliz, Senior Member, IEEE, Olli-Heikki Huttunen, Elina Jansson, Juhani Kemppainen, Jyrki \\ Schroderus, Marika Kurkinen, Tapio Fabritius, Member, IEEE
}

\begin{abstract}
Flexible electrodes are crucial for the growth of modern flexible electronics or smart clothing. Their ability to serve as sensing elements, deliver energy or transmit electrical signals in different conditions is essential for the evolution of wearables that will enrich our everyday life. In this study, we focus our efforts on understanding the impact of an additional protective layer on elongated and washed roll-to-roll printed (R2R) conductive flexible electrodes of various shapes. The electrical and morphological examinations provide information on the performance of the electrodes and materials during washing and elongation cycles. The acquired results indicate propitious electrode shapes as well as data concerning micro-scale cracking mechanisms, which are responsible for electrode performance degradation. The application of commercially available pastes and $R 2 R$ printing methods allow the flawless utilization of the results and the fabrication of flexible and more reliable electronic components.
\end{abstract}

\section{INTRODUCTION}

Compared to conventional "inflexible" electronics, flexible electronics provides new, extraordinary applications and novel integration methods, endorsing the concept of smart clothing and the Internet of Things (IoT) [1], [2].

The aspect of flexibility is especially attractive for sensing applications and health monitoring, where the sensing elements are placed directly onto the skin or embedded into clothing [3]. The capability to embed conductive sensing elements directly into textiles opens new opportunities in the design of e-clothing that continuously monitors the surrounding environment and notifies about anomalies like radiation, illumination changes, toxic chemicals and gases [4]-[6]. Flexibility is also attractive for energy harvesting systems, enabling the application of different energy harvesting methods (e.g., outdoor and indoor photovoltaics, mechanical, and thermostatic) [7][10]. Another important implementation is energy storage, where supercapacitors or batteries, in addition to being flexible, provide necessary energy to supply portable systems [11]. Comprehensive wearable systems would require several different sub-systems equipped with different elements of identification, visualization and communication [12]-[17]. A genuinely portable system

R.S. \& T.F are with the OPEM Unit, ITEE, University of Oulu, Erkki Koiso Kanttilankatu 3, 90570, Oulu, Finland. Corresponding author: R.S. Phone: +358409634100, e-mail: rafal.sliz@oulu.fi.

O.H., E.J. \& M.K. are with VTT Technical Research Center, Kaitovayla 1, 90570, Oulu, Finland.

J.K. \& J.S. are with Polar Electro, Professorintie 5, 90440, Kempele, Finland. would be built of flexible elements connected with rigid conventional electronic parts like processors, ICs, memory and radio transponders. The more flexible elements in the system, the more autonomous, auspicious, and useradaptable the system is, fulfilling the objectives of smart clothing applications [18].

Regardless of the application, all systems need conductive elements either for sensing, data transmission, or to provide energy [19]. Hence, it is crucial for flexible electronics development to advance reliable conductive electrodes and interconnections [20], [21].

Among the many techniques of manufacturing conductive elements, printing is one of the most promising due to the easy and inexpensive implementation, variety of materials and substrates, and thanks to R2R compatibility, high upscaling potential [22], [23]. The ability to utilize various materials is crucial from the sustainability perspective, where rare earth materials and plastics can be exchanged by eco-friendly substitutes such as graphene or nanocellulose [24]-[26].

There have been numerous studies to identify, explain and diminish the breaking mechanisms that are responsible for the reduced reliability of flexible conductive lines [27][29]. Existing research recognizes three main schemes aiming to increase the reliability and performance of the conductive electrodes [30]. The first one incorporates a material composition-oriented methods where conductive nanoparticles/nanowires, combined with elastomers, offer the anticipated level of elasticity, usually at a cost of higher resistivity due to the reduced conductivity of the elastic compounds [31]. The second scheme incorporates various structural configurations with the conductive lines of different shapes providing the capability to elongate the line without breaking the conductive path [32]. The third scheme utilizes an idea of protecting the electrodes with the protective encapsulation layer by embedding them in supportive elastomeric materials [33], [34]. Many researchers combine different strategies to reach the most reliable flexible elements [35].

In this investigation, we focused our efforts on comprehending the influence of an additional conductive/protective layer on the performance of various shaped R2R-printed electrodes during elongation and washing. To ensure that the results can be used by printed and flexible electronics engineers, the presented testing framework includes different conductive inks and electrode shapes (Figure 1a). Since the ultimate objective of this study was to develop a system that can be used for EEG and ECG monitoring (Figure 1b), one of the 
requirements was that the electrodes had to be embedded in the textiles and in contact with skin surface. Therefore, to allow the acquisition of vital signals, the protective layer needed to be conductive. During the elongation cycling, we used resistance as the main indicator of the conductive line performance (Figure 2 \& 3 ). Consequently, we conducted a Scanning Electron Microscope (SEM) assessment of the printed electrodes to identify the breaking process and impact of elongation on the electrode's performance. Finally, we compared our results with the single-layer electrodes and explained these results.

To extend the applicability of the results, in addition to elongation processing, we studied the influence of washing on the behavior of the printed electrodes. Washing cycling is crucial because it involves several mechanical factors that influence the electrodes (i.e., stretching, bending, abrasion, shearing, flexing, and soaking) [36]. Also, the presence of detergents and water accelerates the process of degradation [37]. There have been numerous studies to investigate the influence of washing on printed electrodes [38]-[42]. Nonetheless, there is a number of gaps and shortcomings, especially related to understanding the electrode electrical and surface degradation that occurs during washing.

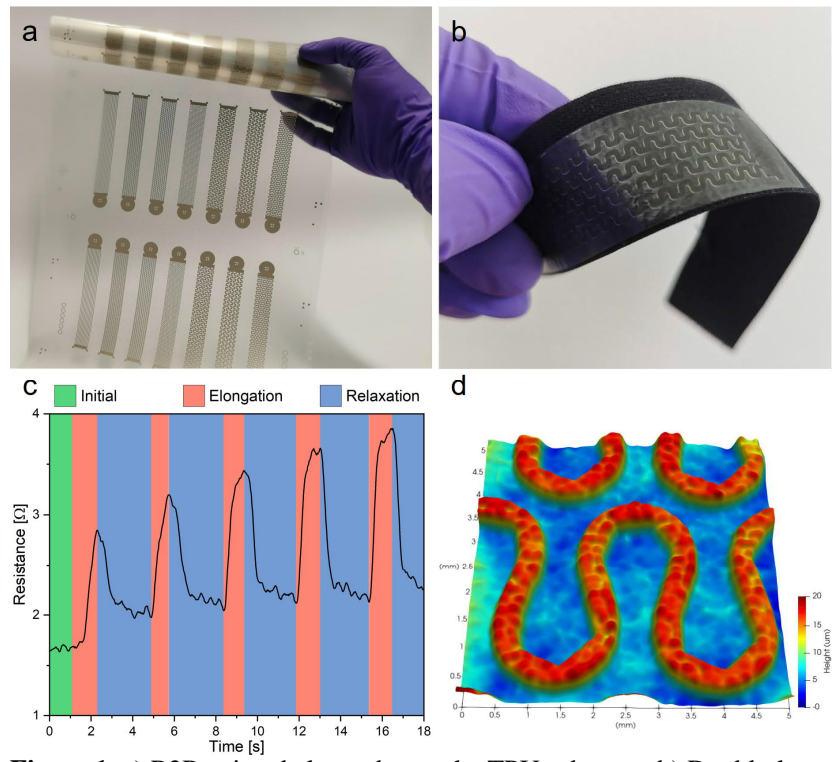

Figure 1. a) R2R-printed electrodes on the TPU substrate. b) Double-layer electrode printed on TPU and attached to the fabric belt prior to the washing tests. c) The electrode resistance changes during the elongation test. d) Surface morphology of the printed electrode.

\section{Methods}

Due to significant web tension during R2R printing, the thermoplastic polyurethane (TPU) substrate needed to be supported with an inelastic carrier. We selected PET due to its suitable mechanical properties and thermal stability. The aspect of thermal stability was especially important for the annealing and plasma-cleaning processes, where the substrate is exposed to excessive thermal stresses. After transferring the TPU (UO73) to the PET carrier, the surface was plasma treated to remove contamination and improve the printing properties [43]. Rotary screen printing was used to print the electrodes of various patterns (Figure 1a). Two different pastes (ECM CI-1036, ECM CI-4040) were utilized to print the first layer of conductive electrodes. Whereas ECM CI-1036 is a silver conductive paste, ECM CI-4040 is a silver/silver chloride conductive paste. After printing, hot air annealing was performed in a convection oven with a temperature of 120 ${ }^{\circ} \mathrm{C}$ for 120 seconds. This step was followed by another 120 seconds at $120{ }^{\circ} \mathrm{C}$ to assure appropriate curing. Consequently, the second conductive/protective layer of the same pattern was printed using ECM CI-2051, which is a stretchable carbon-based paste. The same annealing steps were applied as for the first conductive layer. The layers were printed using a rotary screen - Stork with steel mesh 305/in. with a rate of $2 \mathrm{~m} / \mathrm{min}$.

The resistance during the elongation was measured employing the four-probe measurement system. 10,000 cycles of elongation of $10 \%$ and a speed of one cycle per 2 seconds were applied to each sample. The system used an Agilent 34401A multimeter linked to the computer for control and data acquisition.

For the washing analysis, the electrodes were printed on the TPU substrate and consequently attached to stretchable textile belts (Figure 1b). A standard home washing machine (Siemens WD14H540DN) and the mixed fabrics $\left(40{ }^{\circ} \mathrm{C} \& 1,000 \mathrm{rpm}\right.$ spinning) program were utilized during tests. The washing cycle was repeated 10 times. During washing, the belts were in a washing bag. A NeoScope JCM-5000 system was used to acquire SEM images. The surface morphology measurement of the electrodes (Figure 1d) was performed using an optical profilometer (Bruker ConturGT).

\section{RESULTS}

We used resistance to evaluate the quality of the conductive lines and their performance during elongation and reliability in washing. The initial measurements provided evidence on how the resistivity of the electrodes varies during a short period within the elongation cycle (Figure 1c). The distinct elongation cycle is composed of two main phases: - where the resistance increases due to the electrode stress (elongation), - where the resistance decreases because the electrode returns to its initial state (relaxation). Importantly, with each cycle, the resistance slightly increases. This continuous trend of the increasing resistance and its origins have been elaborated in the previous studies of Liang et al. [44].

Before initiating the elongation cycle, we measured the resistance of the electrodes that were printed with ECM CI-1036 and ECM CI-4040 pastes, and protected with printed ECM CI-2051 paste (Table 1 - untreated samples). 


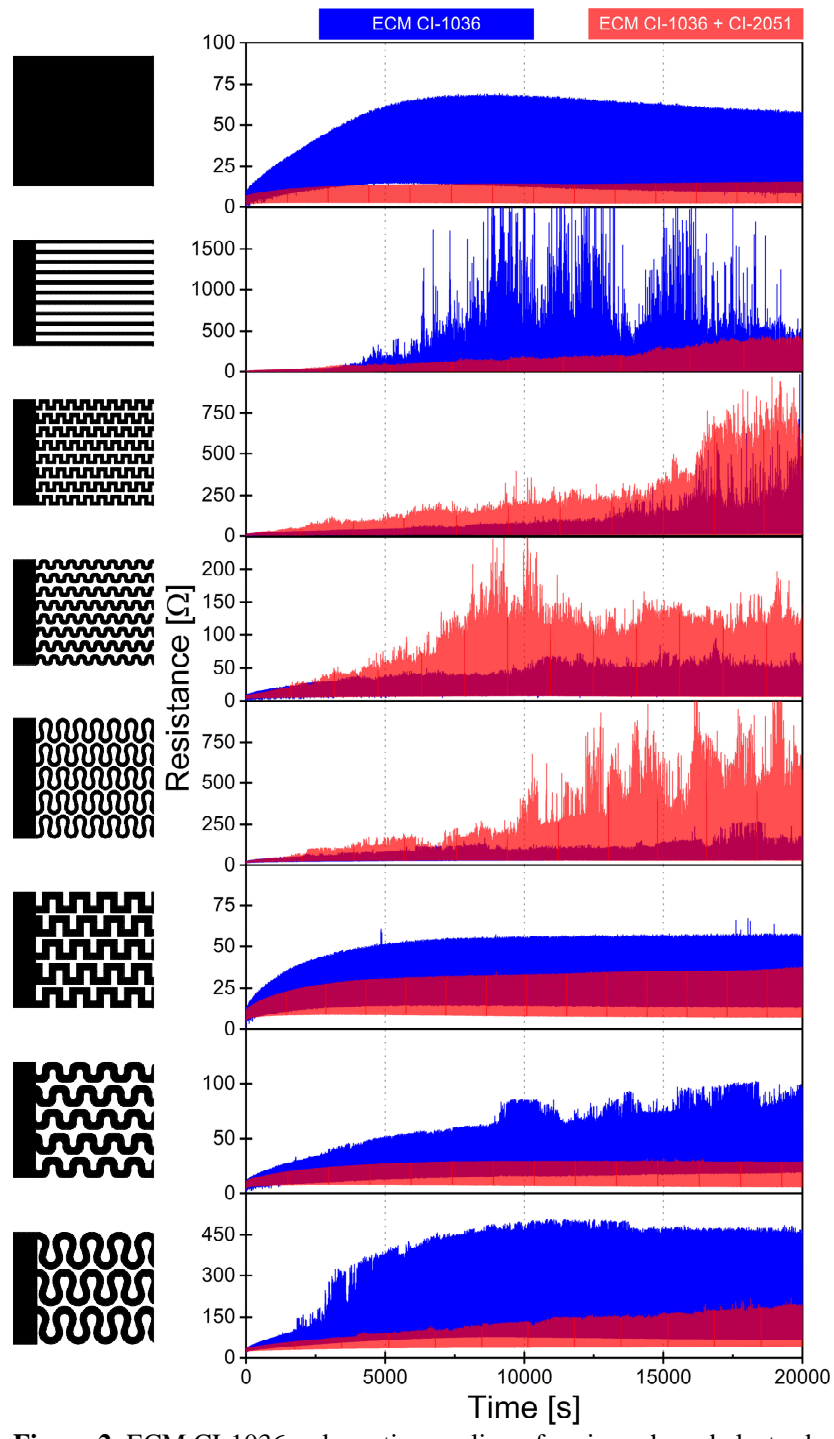

Figure 2. ECM CI-1036 - elongation cycling of various shaped electrodes without (blue) and with a (red) protective layer of ECM CI-2051 paste. The left images refer to the respective shape of the electrode.

Figure 2 shows a comparison of the elongation reliability of single-layer (ECM CI-1036 (blue)) and double-layer (ECM CI-1036 + ECM CI-2051 (red)) conductive electrodes. The single-layer electrode behavior is provided for reference. A similar logic was applied in Figure 3, where the single-layer of ECM CI-4040 (blue) electrodes was compared with a double-layer of ECM CI-4040 + ECM CI-2051 (red).

In the case of ECM CI-1036 (Figure 2), the full coverage, straight lines, and large shape electrodes benefit greatly from the second protective layer, and indicate lower resistance and more stable behavior throughout cycling. At the same time, the small shapes seem to suffer because of the additional protective layer, having higher resistance and less stable behavior. Furthermore, the small-shape double-layer electrodes have larger difference in resistance between elongation and relaxation states, compared to the remaining electrodes.

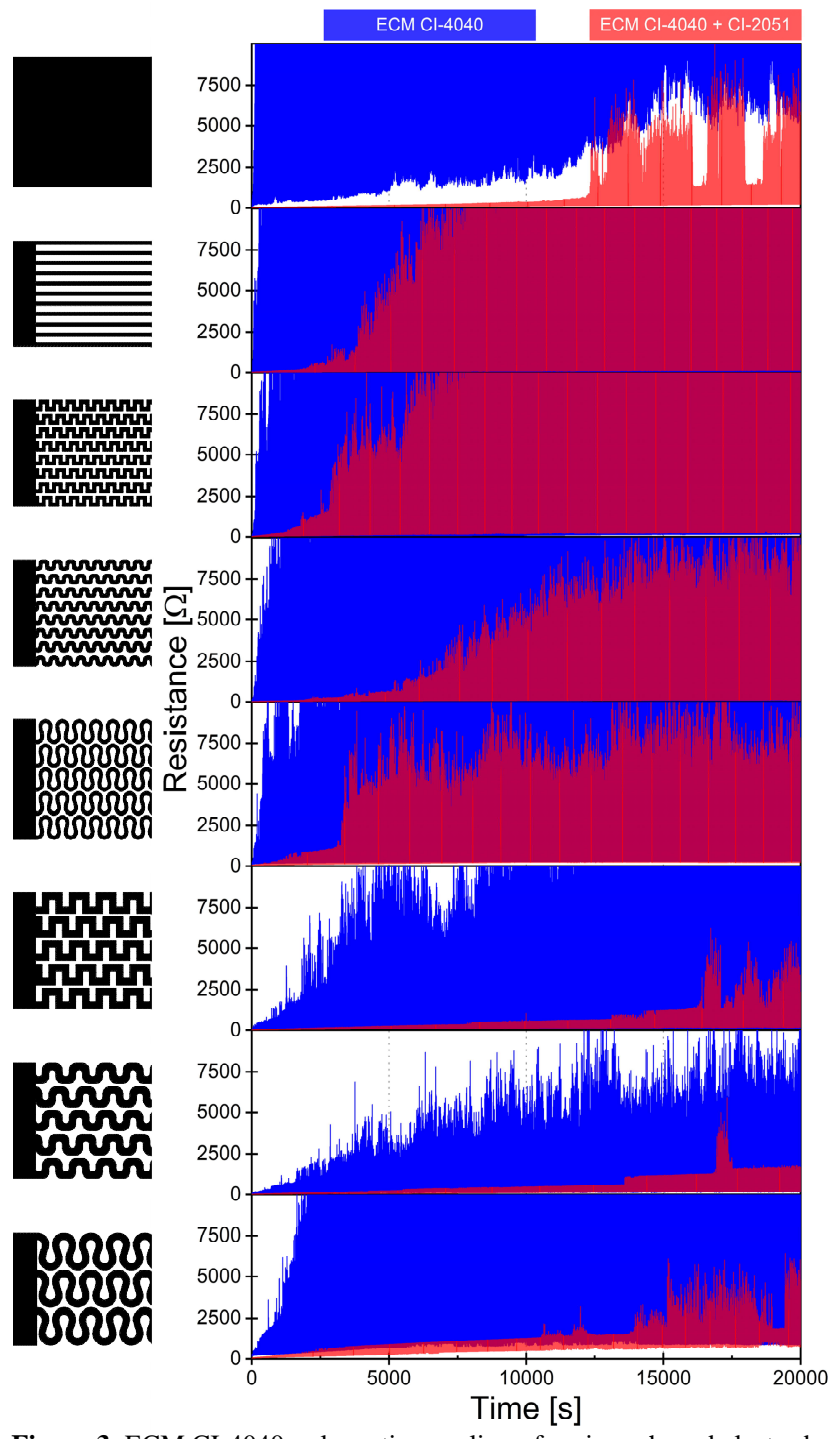

Figure 3. ECM CI-4040 - elongation cycling of various shaped electrodes without (blue) and with a (red) protective layer of ECM CI-2051 paste. The left images refer to the respective shape of the electrode.

For ECM CI-4040 (Figure 3), the positive influence of the protective layer is visible for all shapes of the electrodes reduced resistance compared to single-layer electrodes. In this case, the full coverage and large shapes benefit the most from the protective layer, demonstrating significantly reduced resistance and more stable behavior.

The comparison of ECM CI-1036 and ECM CI-4040 shows that the first has better endurance, regardless of the presence of the protective layer. Consequently, we conducted SEM assessment of the electrodes that had undergone the washing and elongation cycling, and compared the images with the pristine, untreated samples. For ECM CI-1036 (Figure 4), the surface of the singlelayer (unprotected) sample after elongation does not change considerably. For the double-layer (protected) sample, small inconsistencies in the surface morphology are visible in the form of protruding chips. For the washed samples, while the cracks for the unprotected surface are visible, the protected one demonstrates a rather smooth 
surface, without voids and cracks. However, the signs of washed away material are visible.

SEM images of ECM CI-4040 (Figure 5), show that for the single-layer elongated sample, the surface is reasonably smooth without cracks and void. At the same time, the double-layer elongated sample show signs of cracking on the surface. For the washed samples of ECM CI-4040, while the single-layer sample has cracks and voids, the protected one looks smooth with insignificant signs of a washed-off surface. In terms of washing reliability, ECM CI-4040 behaves similarly to ECM CI1036.

Finally, the results shown in Table 1 demonstrate the disparity between washed and unwashed electrodes. In case of full coverage electrodes, washing causes a drastic increase of the resistance. We assume that the increase of the resistance is caused by the accelerated washing of conductive carbon particles from the protective layer.

Table 1. The resistance of various shaped electrodes (horseshoe and full coverage) before and after the washing cycles

\begin{tabular}{|c|c|c|c|c|}
\hline & Unwashed & Washed & Unwashed & Washed \\
\hline $\begin{array}{l}\text { CI-1036 } \\
\text { CI-2051 }\end{array}$ & $1.55 \pm 0.68 \Omega$ & $53.1 \pm 23.3 \mathrm{k} \Omega$ & $25.44 \pm 4.38 \Omega$ & $221.0 \pm 25.8 \mathrm{k} \Omega$ \\
\hline \begin{tabular}{l|} 
CI-4040 \\
CI-2051
\end{tabular} & $3.12 \pm 0.82 \Omega$ & $123.4 \pm 46.3 \mathrm{k} \Omega$ & $31.86 \pm 1.13 \Omega$ & $234.0 \pm 25.8 \mathrm{k} \Omega$ \\
\hline
\end{tabular}

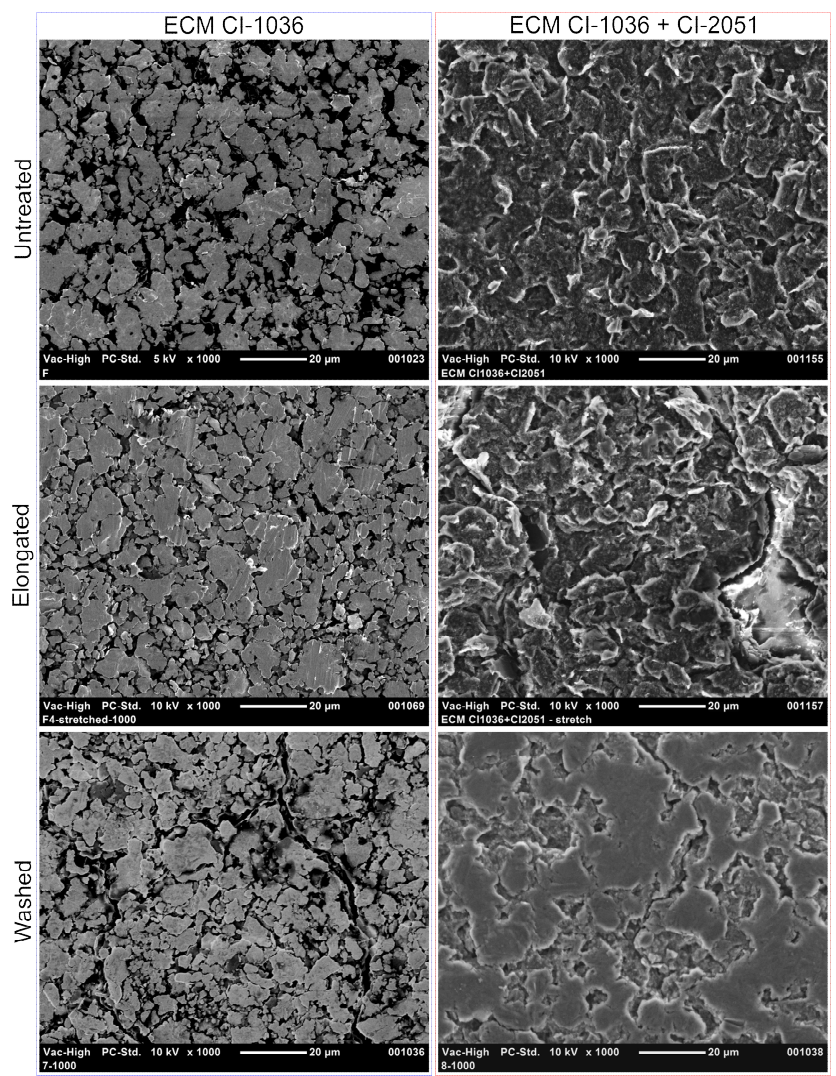

Figure 4. ECM CI-1036: SEM images of untreated electrodes - top row, elongated electrodes - middle row, washed electrodes - bottom row.

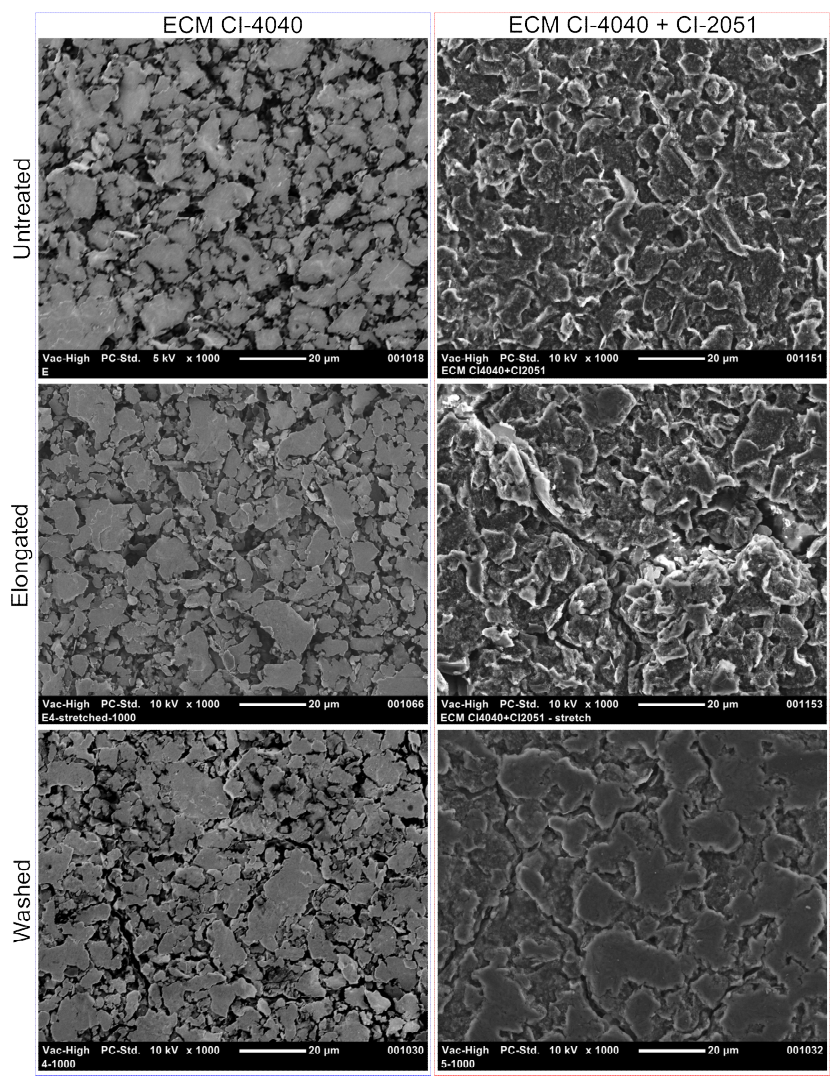

Figure 5. ECM CI-4040: SEM images of untreated electrodes - top row, elongated electrodes - middle row, washed electrodes - bottom row.

\section{Discussion AND CONCLUSIONS}

The main aim of this experimental research was to comprehend how the additional layer of conductive material protects the electrodes of various shapes and inks, and provide a guideline that can be used for creating flexible ecosystem for humans' vital signs measurements. Hence, as a protective layer, we used a material that has conductive properties, ECM CI-2051.

The resistance variation acquired during experiments points to a few aspects that need further explanation.

The additional protective layer printed on top of the electrode serves a double purpose: it protects the layer beneath and enhances the electrical properties of the electrodes. As seen in Figures $2 \& 3$, in most cases the resistance of the double-layer electrodes decreases, and the reliability increases. SEM images indicate a presence of cracks and voids on the surface of the protected electrodes that underwent the elongation cycling. After considering the resistance values, we attribute the increased amount of cracking on the surface to the electrodes' increased thickness. Although the cracks are visible, most probably they affect only the top layer, protecting the layer beneath. The shape analysis suggests that larger structures better withstand the elongation. One of the reasons is the reduced width and radius of the smaller patterns, which are more significantly affected by the same degree of elongation. These results are in agreement with the previous research [45], [46]. 
The washing tests show how important are the aspects of detergent and water. The SEM images of unprotected electrodes indicate that water rinses away the conductive material leaving voids and cracks that contribute to the electrodes' higher resistance. The results shown in Table 1 imply that washing is more destructive for the unprotected electrodes, regardless of shape or material. The results indicate the necessity for more research concerning the washing of flexible electronics elements, as this process is particularly destructive.

To conclude, this research provides evidence regarding the influence of the protective conductive layer on the performance of R2R-printed electrodes subjected to elongation and washing cycling. The results indicate the positive impact of the protective layers and suggest shapes and patterns that are optimal for implementation in smart clothing applications.

\section{REFERENCES}

[1] Y. Zhan, Y. Mei, and L. Zheng, "Materials capability and device performance in flexible electronics for the Internet of Things," $J$. Mater. Chem. C, vol. 2, no. 7, pp. 1220-1232, 2014.

[2] A. Nathan et al., "Flexible electronics: The next ubiquitous platform," Proc. IEEE, vol. 100, no. SPL CONTENT, pp. 14861517,2012

[3] S. Huang, Y. Liu, Y. Zhao, Z. Ren, and C. F. Guo, "Flexible Electronics: Stretchable Electrodes and Their Future," Adv. Funct Mater., vol. 29, no. 6, pp. 1-15, 2019.

[4] E. Singh, M. Meyyappan, and H. S. Nalwa, "Flexible GrapheneBased Wearable Gas and Chemical Sensors," ACS Appl. Mater. Interfaces, vol. 9, no. 40, pp. 34544-34586, 2017.

[5] J. Kukkola et al., "Novel printed nanostructured gas sensors," Procedia Eng., vol. 25, pp. 896-899, 2011.

[6] R. Sliz et al., "Stable Colloidal Quantum Dot Inks Enable InkjetPrinted High-Sensitivity Infrared Photodetectors," ACS Nano, vol 13, pp. 11988-11995, 2019.

[7] B. J. Kim et al., "Highly efficient and bending durable perovskite solar cells: Toward a wearable power source," Energy Environ. Sci., vol. 8, no. 3, pp. 916-921, 2015.

[8] H. Wu, Y. A. Huang, F. Xu, Y. Duan, and Z. Yin, "Energy Harvesters for Wearable and Stretchable Electronics: From Flexibility to Stretchability," Adv. Mater., vol. 28 , no. 45, pp. 9881-9919, 2016.

[9] R. Sliz, Y. Suzuki, T. Fabritius, and R. Myllyla, "Influence of temperature on wetting properties of thin films in organic solar cells applications," Colloids Surfaces A Physicochem. Eng. Asp., vol. 443, pp. 182-187, 2014.

[10] M. Ylikunnari et al., "Flexible OPV modules for highly efficient indoor applications," Flex. Print. Electron., vol. 5, p. 014008, Jan. 2020.

[11] A. M. Gaikwad, A. C. Arias, and D. A. Steingart, "Recent Progress on Printed Flexible Batteries: Mechanical Challenges, Printing Technologies, and Future Prospects," Energy Technol., vol. 3, no. 4, pp. 305-328, 2014.

[12] S. Choi et al., "Highly Flexible and Efficient Fabric-Based Organic Light-Emitting Devices for Clothing-Shaped Wearable Displays," Sci. Rep., vol. 7, no. 1, pp. 1-8, 2017.

[13] K. Jokinen, A. Bykov, R. Sliz, K. Remes, T. Fabritius, and R. Myllylä, "Luminescence and spectrum variations caused by thermal annealing in undoped and doped polyfluorene OLEDs," Solid. State. Electron., vol. 103, pp. 184-189, 2015.

[14] P. Vilmi, S. Varjo, R. Sliz, J. Hannuksela, and T. Fabritius, "Disposable optics for microscopy diagnostics," Nat. Publ. Gr., pp. $2-7,2015$.

[15] R. S. Deol, H. W. Choi, M. Singh, and G. E. Jabbour, "Printable displays and light sources for sensor applications: A review," IEEE Sens. J., vol. 15, no. 6, pp. 3186-3195, 2015.
[16] E. Abad et al., "Flexible tag microlab development: Gas sensors integration in RFID flexible tags for food logistic," Sensors Actuators, B Chem., vol. 127, no. 1, pp. 2-7, 2007.

[17] Z. Xie, R. Avila, Y. Huang, and J. A. Rogers, "Flexible and Stretchable Antennas for Biointegrated Electronics," Adv. Mater., vol. 1902767, pp. 1-16, 2019.

[18] J. Kim et al., "Miniaturized Flexible Electronic Systems with Wireless Power and Near-Field Communication Capabilities," Adv. Funct. Mater., vol. 25, no. 30, pp. 4761-4767, 2015.

[19] N. Matsuhisa et al., "Printable elastic conductors with a high conductivity for electronic textile applications," Nat. Commun., vol. 6, no. May, p. 7461, 2015.

[20] J. Perelaer et al., "Printed electronics: The challenges involved in printing devices, interconnects, and contacts based on inorganic materials," J. Mater. Chem., vol. 20, no. 39, pp. 8446-8453, 2010.

[21] R. Sliz et al., "Reliability of R2R-printed, flexible electrodes for eclothing applications," npj Flex. Electron., no. 4, 2020.

[22] O. H. Huttunen, T. Happonen, J. Hiitola-Keinänen, P. Korhonen, J. Ollila, and J. Hiltunen, "Roll-To-Roll Screen-Printed Silver Conductors on a Polydimethyl Siloxane Substrate for Stretchable Electronics," Ind. Eng. Chem. Res., vol. 58, no. 43, pp. 19909 19916, 2019.

[23] H. W. Choi, T. Zhou, M. Singh, and G. E. Jabbour, "Recent developments and directions in printed nanomaterials," Nanoscale, vol. 7, pp. 3338-3355, 2015.

[24] F. Hoeng, A. Denneulin, and J. Bras, "Use of nanocellulose in printed electronics: A review," Nanoscale, vol. 8, no. 27, pp. 13131-13154, 2016.

[25] W. J. Hyun, E. B. Secor, M. C. Hersam, C. D. Frisbie, and L. F. Francis, "High-resolution patterning of graphene by screen printing with a silicon stencil for highly flexible printed electronics," Adv. Mater., vol. 27, no. 1, pp. 109-115, 2015.

[26] R. Hartmann et al., "Interactions between aminated cellulose nanocrystals and quartz: Adsorption and wettability studies," Colloids Surfaces A Physicochem. Eng. Asp., vol. 489, pp. $207-$ 215, 2016.

[27] A. J. Baca et al., "Semiconductor wires and ribbons for highperformance flexible electronics," Angew. Chemie - Int. Ed., vol. 47, no. 30, pp. 5524-5542, 2008.

[28] J. A. Rogers, T. Someya, and Y. Huang, "Materials and mechanics for stretchable electronics," Science (80-. )., vol. 327, no. 5973, pp. $1603-1607,2010$

[29] Y. Ma, X. Feng, J. A. Rogers, Y. Huang, and Y. Zhang, "Design and application of 'J-shaped' stress-strain behavior in stretchable electronics: A review," Lab Chip, vol. 17, no. 10, pp. 1689-1704, 2017.

[30] D. H. Kim and J. A. Rogers, "Stretchable electronics: Materials strategies and devices," Adv. Mater., vol. 20, no. 24, pp. 48874892,2008

[31] Y. I. Lee, S. Kim, S. B. Jung, N. V. Myung, and Y. H. Choa, "Enhanced electrical and mechanical properties of silver nanoplatelet-based conductive features direct printed on a flexible substrate," ACS Appl. Mater. Interfaces, vol. 5, no. 13, pp. 59085913, 2013.

[32] J. A. Fan et al., "Fractal design concepts for stretchable electronics," Nat. Commun., vol. 5, pp. 1-8, 2014.

[33] K. Li et al., "A Generic Soft Encapsulation Strategy for Stretchable Electronics," Adv. Funct. Mater., vol. 29, no. 8, pp. 1$12,2019$.

[34] F. Bossuyt, T. Vervust, and J. Vanfleteren, "Stretchable electronics technology for large area applications: Fabrication and mechanical characterization," IEEE Trans. Components, Packag. Manuf. Technol., vol. 3, no. 2, pp. 229-235, 2013.

[35] K. D. Harris, A. L. Elias, and H. J. Chung, "Flexible electronics under strain: a review of mechanical characterization and durability enhancement strategies," J. Mater. Sci., vol. 51, no. 6, pp. 2771-2805, 2016.

[36] S. U. Zaman, X. Tao, C. Cochrane, and V. Koncar, "Market readiness of smart textile structures - reliability and washability," IOP Conf. Ser. Mater. Sci. Eng., vol. 459, p. 012071, Dec. 2018.

[37] E. Ismar, S. uz Zaman, X. Tao, C. Cochrane, and V. Koncar, "Effect of Water and Chemical Stresses on the Silver-Coated Polyamide Yarns," Fibers Polym., vol. 20, no. 12, pp. 2604-2610, 2019. 
[38] A. Achilli, D. Pani, and A. Bonfiglio, "Characterization of screenprinted textile electrodes based on conductive polymer for ECG acquisition," Comput. Cardiol. (2010)., vol. 44, pp. 1-4, 2017.

[39] P. C. Hsu, C. L. Shen, F. L. Chen, H. S. Huang, W. C. Wang, and T. H. Huang, "A printed physiological monitoring module in etextile," 2018 Int. Flex. Electron. Technol. Conf. IFETC 2018, pp. $1-2,2018$.

[40] A. Achilli, A. Bonfiglio, and D. Pani, "Design and characterization of screen-printed textile electrodes for ECG monitoring," IEEE Sens. J., vol. 18, no. 10, pp. 4097-4107, 2018.

[41] D. J. Preston et al., "Heat Transfer Enhancement during Water and Hydrocarbon Condensation on Lubricant Infused Surfaces," Sci. Rep., vol. 8, no. 1, pp. 1-9, 2018.

[42] M. J. Kye et al., “'Drop-on-textile' patternable aqueous PEDOT composite ink providing highly stretchable and wash-resistant electrodes for electronic textiles," Dye. Pigment., 2018.

[43] R. Sliz, Y. Suzuki, A. Nathan, R. Myllyla, and G. Jabbour, "Organic solvent wetting properties of UV and plasma treated $\mathrm{ZnO}$ nanorods: printed electronics approach," Org. Photovoltaics XIII, vol. 8477 , p. $84771 \mathrm{G}, 2012$

[44] J. Liang et al., "Silver Nanowire Percolation Network Soldered with Graphene Oxide at Room Temperature and Its Application for Fully Stretchable Polymer Light-Emitting Diodes," ACS Nano, vol. 8, no. 2, pp. 1590-1600, Jan. 2014.

[45] H. Hocheng and C. M. Chen, "Design, fabrication and failure analysis of stretchable electrical routings," Sensors, vol. 14, no. 7 pp. 11855-11877, 2014.

[46] B. Marchiori, R. Delattre, S. Hannah, S. Blayac, and M. Ramuz, "Laser-patterned metallic interconnections for all stretchable organic electrochemical transistors," Sci. Rep., vol. 8, no. 1, pp. 19, 2018 . 